\title{
Quantitative 3D Characterization of Cellular Materials: Segmentation and Morphology of Foam
}

\author{
Kevin Mader, ${ }^{1,2}$ Rajmund Mokso, ${ }^{1}$ Christophe Raufaste,${ }^{3}$ Benjamin \\ Dollet, ${ }^{4}$ Stéphane Santucci, ${ }^{5}$ Jérôme Lambert, ${ }^{4}$ and Marco Stampanoni ${ }^{1,2}$ \\ ${ }^{1}$ Swiss Light Source, Paul Scherrer Institut, Villigen, Switzerland \\ ${ }^{2}$ Institute for Biomedical Engineering, University and ETH Zurich, Gloriastrasse 35, Zurich, Switzerland \\ ${ }^{3}$ Laboratoire de Physique de la Matière Condensée, \\ UMR 7336 CNRS and Université Nice-Sophia Antipolis, Parc Valrose, F-06108 Nice Cedex 2, France \\ ${ }^{4}$ Institut de Physique de Rennes, UMR 6251 CNRS/Université de Rennes 1, \\ Campus Beaulieu, Bâtiment 11A, 35042 Rennes Cedex, France \\ ${ }^{5}$ Laboratoire de Physique, ENS Lyon, UMR CNRS 5672, 46 allée d'Italie, 69007 Lyon, France
}

Wood, trabecular bone, coral, liquid foams, grains in polycrystals, igneous rock, and even many types of food share many structural similarities and belong to the general class called cellular materials. The visualization of these materials in $3 \mathrm{D}$ has been made possible in the last decades through a variety of imaging techniques including magnetic resonance imaging (MRI), micro-computed Xray tomography $(\mu \mathrm{CT})$, and confocal microscopy. Recent advances in synchrotron-based ultra fast tomography have enabled measurements in liquid foams with thousands of bubbles and time resolutions down to 0.5 seconds. Post-processing techniques have, however, not kept pace and extracting useful physical metrics from such measurements is far from trivial. In this manuscript we present and validate a new, fully-automated method for segmenting and labeling the void space in cellular materials where the walls between cells are not visible or present. The individual cell labeling is based on a new tool, the Gradient Guided Watershed, which, while computationally simple, can be robustly scaled to large data-sets. Specifically we demonstrate the utility of this new method on several liquid foams (with varying liquid fraction and polydispersity) composed of thousands of bubbles, and the subsequent quantitative 3D structural characterization of those foams. 


\section{INTRODUCTION}

Dispersions, which by definition consist of at least two phases mixed together, are an interesting class of materials, principally because the mechanical properties of a mixture are not a linear sum of its components. Furthermore these materials are particularly useful because their properties can be tuned by adjusting the relative composition, size distribution, and topology rather than their chemical make-up. For this reason, they are widely seen in nature as wood and coral, and in synthetic materials such as metallic and liquid foams, and a multitude of ceramic, glass, and even food products. Many of these materials can be described as cellular, meaning one of the phases is made up of individual cells or units packed together. The use of a cellular model provides a powerful framework for characterizing and understanding the physical, rheological, and mechanical properties of a material.

Experimentally it has always been difficult to access the inner 3D structure of cellular materials. The development over the last decades of micro-computed tomography $(\mu \mathrm{CT})$, confocal microscopy, scanning electron microscopy (SEM), and magnetic resonance imaging (MRI) have provided the ability to penetrate into these materials providing valuable images of the internal organization. Translating these images to structure and consequently individual cells is highly variable in both feasibility and difficulty. While numerous specific techniques exist, a majority of the tools are based on closed-source proprietary software packages such as Mavi (Fraunhofer ITWM, Kaiserslautern, Germany), Avizo (Visualization Sciences Group, Burlington, USA), or Aphelion 3D (ADCIS SA, Saint-Contest, France). These packages, while generally polished and powerful, limit the flexibility and scalability of the analysis being done. Furthermore they make it difficult to understand and improve on existing methods as certain functions are effectively black boxes.

Liquid foam is a cellular material of particular interest because of its wide industrial applicability and thus chosen for our further analysis. The structure of liquid foams consists of gas bubbles dispersed inside a continuous liquid phase [1]. The bubbles are separated by a thin layer of liquid called a film and the liquid built up when more than 2 bubbles touch is called a Plateau border. Owing to this multiphasic composition, they exhibit interesting mechanical properties that lead to numerous applications in food and cosmetic industries and are used to optimize ore and oil extraction. Experimentally imaging foams can be difficult; in dry foams, most of the liquid accumulates at the junction of thin films, forming a continuous network of liquid channels called Plateau borders [1, 2]. Films and Plateau borders absorb light weakly but diffuse it strongly, making the structure opaque and difficult to image using visible light and other standard approaches. Several alternative more sophisticated techniques have been therefore proposed to image 3D foams in depth. Magnetic-resonance imaging, for example, has successfully been used to visualize 200 bubbles in [3], as opposed to 48 obtained with optical tomography in [4]. X-ray tomography has also been applied to the examination of liquid foams; early results in 2005 successfully scanned 750 bubbles with an acquisition time of $150 \mathrm{~s}$ [5]. Setup optimization has led to a steady improvement to 30000 bubbles in $30 \mathrm{~s}$ by 2010 in [6], enabling the observation of the very slow evolution processes in coarsening foams. More recently improvements in detectors have enabled scans to be conducted $60 \times$ faster with the same resolution and field of view with only marginal losses in image quality [7].

When probing dry $(<10 \%$ liquid fraction) liquid foams with hard X-rays (wavelength $<0.1 \mathrm{~nm})$ thin films $(<1 \mu \mathrm{m})$ interact or absorb weakly the X-ray light, making them, in general, impossible to distinguish from background noise. Plateau borders, being much thicker, absorb and diffuse more light and provide a clear contrast from the air-filled bubbles (Fig. 1a). The step between Plateau borders visualization (Fig. 1b) to individually, uniquely labeled bubbles is far from trivial. Earlier studies [6, 8] have successfully labeled bubbles based on X-ray tomographic images, but relied on proprietary software with limited flexibility and required subdividing the field of view [5] making tasks such as sensitivity analyses and tracking much more difficult. More recently another group also produced a good labeling of monodisperse liquid foam data [9], but is again dependent on proprietary commercial software.

The rapid improvements in acquisition time come at some cost in the form of decreased image contrast and increased background noise and motion artifacts necessitating sensitive and noise resistant post-processing techniques, which can be easily adapted to the quality of the images at hand. Furthermore the 4D multi-gigabyte data sets acquired require efficient, scalable, hands-free methods for analyzing these data. The tools described in this manuscript are an effort to satisfy the needs in this community for automated quantitative analysis of large datasets with special emphasis on complex liquid foams systems where the presently available tools fail to provide open, scalable, reliable and consistent results.

Our approach is similar in principle to the watershed transform [10-12], but more adaptable to a variety of problems and volume fractions while providing tunable parameters, which can be adjusted based on the information content of the data, specifically, noise-level, volume fraction, and contrast. The watershed transform was also the basis for the analysis peformed in [8], but required proprietary software and functions to create a usable labeling. Consequently 
it would be difficult to further scale and adapt to new sorts of problems. The methods and tools described in this manuscript open numerous possibilities in the Materials Science community. They are tested and validated on liquid foams samples, but have been built in a generic way so they can be directly used for labeling and analysis of other cellular materials, such as wood or the trabecular structure of bone.

\section{MATERIALS AND METHODS}

\section{Sample Preparation}

We prepared several samples of liquid foams. FOAM-M and FOAM-P were made of the mixture of sodium lauryl ether sulfate (SLES), cocamidopropyl betaine (CAPB) and myristic acid (MAc), following the protocol proposed by [13]: we prepared a solution of $6.6 \%$ of SLES and $3.4 \%$ of CAPB in mass in ultrapure water; we then dissolved $0.4 \%$ in mass of MAc by stirring and heating at $60^{\circ} \mathrm{C}$ for about one hour, and we diluted 20 times this solution. A few $\mathrm{mL}$ of solution was poured in the bottom of a cylindrical plexi-glass container with a diameter of $22 \mathrm{~mm}$ and the height of $50 \mathrm{~mm}$ compatible with 180 degree tomography. The foam FOAM-M was prepared by injecting air through a nozzle of diameter $0.4 \mathrm{~mm}$ immersed in the solution, at a flow rate of $1 \mathrm{~mL} / \mathrm{min}$ controlled by a syringe pump (PHD-2000, Harvard Apparatus). When building a monolayer with the so-blown bubbles, a crystallized 2D foam appears, suggesting that the bubble volume dispersity is narrow and FOAM-M can be described as monodisperse. The order of magnitude for bubble volume was roughly estimated to be around $0.1 \mathrm{~mm}^{3}$. FOAM-P was prepared by hand shaking the container for about 30 seconds and was visibly more polydisperse than the former sample. Excess of solution was sucked out of the cell by a syringe when necessary. Foam height in the container ranged between 2 and 5 $\mathrm{cm}$. This is much higher than the millimetric size of the capillary length of the solution so that the foam is subjected to a vertical drainage. Nevertheless, this system was selected because it produces very stable foams, irrespective of its interfacial rheological properties. The drainage is slowed down so that no significant evolution is observed during the first 15 minutes following the foam preparation (which never exceeded the duration of our experiments). However, since the transfer to the beam-line and the scanning of the various samples were performed at different times, we expect that our different foam samples have different liquid fraction, due to the slow drainage process. Furthermore due to the sample preparation method, the liquid fraction was difficult to measure directly, thus the displayed quantity has been inferred a posteriori using the ratio of liquid voxels to total voxels in the images.

\section{Imaging Technique}

The samples were measured at the the superbending magnet of the TOMCAT beamline [14] of the Swiss Light Source. A tomographic dataset is composed of individual radiographic projections of the sample at equidistant angular positions between 0 and 180 degrees. A new rapid tomographic data acquisition scheme using filtered polychromatic X-rays and a CMOS detector of 12 bit dynamic range [7] allows the acquisition of a full set of (500-800) tomographic projections in typically 0.5 seconds with the voxel sizes ranging from 0.5 to $11 \mu \mathrm{m}$ and a corresponding field of view of 0.7 to $22 \mathrm{~mm}$. These new capabilities allow the monitoring of the dynamics of liquid froth systems by acquiring a time series of 3D tomographic images.

With sample holder containing the foam mounted on the sample stage 25 meters downstream of the bending magnet, the acquisition of a single tomographic dataset took $0.5 \mathrm{~s}$, with a nominal pixel size of $11 \mu \mathrm{m}$ and the corresponding field of view of $22 \mathrm{~mm}$ in horizontal direction (smaller in the vertical direction because of the smaller X-ray beam only $6 \mathrm{~mm}$ in size). A scintillator screen was used to convert X-rays to visible light, which could then be recorded as individual projections by the CMOS detector. To reconstruct the set of projections to a 3D stack of images, we used an in-house Fast-Fourier-based reconstruction technique called Gridrec which is nearly equivalent to filtered back-projection but many times faster [15]. The resulting volumetric data is $2016 \times 2016 \times 500$ voxels with an isotropic voxel size of $11 \times 11 \times 11 \mu \mathrm{m}$.

\section{A new labeling method}

The measured volumetric data is a 3D gray-leveled image where under ideal circumstances the voxel intensity corresponds to the amount of X-Ray absorption which occurs in that region. Therefore, brighter regions corresponds to vertices and Plateau borders which strongly absorb the X-rays, while the bubbles and their interstitial films, which 
are too thin to be observed, remain darker (Fig. 1a). We describe in this section the different steps of analysis from the tomogram to the labeling of each individual bubbles.

\section{Image segmentation}

The first step applied to the raw tomographic reconstructions is the application of a median filter to smooth the data and remove spike artifacts. Next a threshold is applied to segment the more strongly absorbing Plateau borders from the more weakly absorbing air-filled bubbles (Fig. 1b). For this step to be successful, the input dataset must be of good quality in terms of signal to noise ratio and contrast. The segmentation is then improved by using the morphological operations of erosion and dilation to remove spurious voxels and holes from the image (Plateau borders) and its inverse (bubbles).

\section{Distance map}

The subsequent analysis is done using two voxel masks as input. The first is the segmented collection of Plateau borders in the image to be called PLAT (Fig. 1b). The second is the region where the bubbles and films are, to be called MASK (Fig. 1c). When the sample occupies the entire field of view, these two images are complementary and MASK is simply the inversion of PLAT. Since the acquisition technique provides isotropic spatial information, we formally define our image as an $L \times W \times H$ lattice of touching, non-overlapping cubes. We shall define a voxel as an individual cube from this lattice, $X=(x, y, z)$ and its 26-Neighborhood $N_{26}(X)$ as all the voxels Y which share a face or edge with $X$. From these starting data sets, like in [8] we create a Euclidean distance map based on the voxel-center positions to be called DIST (figure 1d) from PLAT and MASK where the values are generated by calculating for each voxel in MASK the Euclidean distance to the nearest voxel in PLAT [16].

\section{Bubble seeds}

We define bubble seeds as points which can be used to grow bubbles. We calculate the Gradient and the Hessian of the distance (to the nearest plateau borders DIST) for every voxel in the image based on its respective $N_{26}$ neighborhood. We determine which of these voxels are local maxima (in the distance map) by finding voxels where the Gradient norm is below a flatness criterion and the Hessian is finite negative. The flatness criterion is how low the gradient at a point needs to be in order for that point to be eligible to be a bubble seed. A high value means many points are taken as bubble seeds and a lower value means fewer. In order to minimize spurious bubble seeds we impose several additional criteria on the maxima: for our samples we used a minimum bubble radius of 4 voxels resulting from the noise content analysis of the images. Additionally we ignored minima found within 2 voxels of the image edge, since operations involving derivatives require at least two voxels of boundary to be calculated (below and above). These parameters are both due to the discrete nature of data and are not inherently resolution-dependent.

\section{Bubble growth and Gradient Guided Watershed (GGW)}

This step describes how we grow the bubbles from potential bubble seeds. At this step, our method differs signficantly from the existing Watershed-based techniques. Here we use a distance guided dilation process to robustly identify the different bubbles. The Gradient Guided Watershed method works by determining the entire volume for a bubble given a single or group of voxels, which are identified as a bubble seed $(S)$ and the Plateau border distance map (DIST). The process is an iterative dilation with the added constraint that the new voxels must follow the distance gradient. With the watershed algorithm, an analogy is made between the labeling of empty voxels and water (seeds) flowing into different basins (empty voxels) [12]; using a similar analogy, our technique can be seen as the addition of an effective friction, put in place to only allow the water to flow when the gradient is steep enough (larger than $\left.T_{\text {growth }}\right)$. Mathematically this is expressed in terms of a voxel $(X)$ contained in the bubble $(S)$. Given an empty voxel $Y$ within the $N_{26}(X)$ region (bordering voxel $\left.X\right)$, Y is only to be added to bubble $\mathrm{S}$ when $D I S T(Y) \leq D I S T(X)$ and $\left\|\frac{D I S T(Y)-D I S T(X)}{Y-X}\right\| \geq T_{\text {growth }}$.

The voxels, which are added to $S$ during this procedure, are then excluded in the search for the possible next bubble seed. The procedure is repeated in descending order according to local maximum value (approximately bubble radius) until no more satisfactory local maxima are identified. This allows a larger bubble to engulf any local maxima inside it. The results of this dilation is shown on 2D experimental data in Figure 2a.

\section{Final steps}

After each bubble has been labeled using this technique, there is a large amount of unlabeled air (MASK) remaining in the image (Fig. 3a), particularly in the regions where films are; indeed, the method deliberately avoids films since they do not have strong gradients in the distance map field being between two Plateau borders (blue lines in Fig. $2 \mathrm{~b}$ ). These remaining regions are filled by repeating the GGW with a less stringent $T_{\text {growth }}$ parameter called $T_{\text {fill }}$ and finally, optionally, filling the remaining regions using a Voronoi tessellation (Fig. 2a). 


\section{Implementation}

These analyses were done using custom-developed tools written in Java for portability and the availability of existing open-source tools frameworks like ImageJ (National Institutes of Health, Bethesda, Maryland, USA). We provide the source code for the parallel implementation we developed free of charge [17].

\section{VALIDATION OF OUR NEW LABELING METHOD}

We first developed and calibrated our image analysis tools on a series of synthetically generated cellular materials with various known cell positions, and sizes (data not shown). However, in order to fully validate our approach, the segmentation and labeling of real experimental data is required. Thus, we have tested our approach on the two systems described previously, a mono- and a poly-disperse foam, respectively FOAM-M and FOAM-P.

First, we describe in full detail how we determined the set of parameters to correctly reconstruct and label the bubbles of the mono-disperse foam, FOAM-M.

\section{Parameter Selection}

This section describes the procedure required in order to find the best set of parameters to label the bubbles. The following steps make up the calibration procedure for our method and only need to be performed once to ensure the quality of the labeling :

1. Identify a flatness criterion for bubble seeds.

The flatness criterion is how low the gradient at a point needs to be in order for that point to be eligible to be a bubble seed. A high value means many points are taken as bubble seeds and a lower value means fewer (figure 3). We investigated the general effect of the flatness by varying it systematically between the smallest and largest reasonable values for a discrete system (0 and 1). From these results it was possible to choose by visual inspection of the bubbles the physically meaningful subregion between 0.3 and 0.5 for more detailed investigation. We observe in figure $4 \mathrm{c}$ that within the flatness range, the volume distribution presents two well separated peaks. The larger volume one has an average bubble volume of $0.08 \mathrm{~mm}^{3}$ or 70,000 voxels, while the smaller one has a volume smaller than 10,000 voxels. Below a flatness criterion of 0.3 , both groups scale similarly with flatness, because of the fact more and more bubble seeds are grown. Above a flatness criterion of 0.3 , there is a distinct, quantitative change in the trend: while the number of small bubbles continues to increase, the number of large bubbles saturates around a value of 360 (figure 4f). The same holds for the complete volume distribution as well (figure 4c): the larger bubble distribution saturates, while the peak of the smaller bubbles continues to increase. Guided by knowledge of the preparation and visual inspection of the sample, we interpret the larger bubbles as expected, real bubbles. The fact that their number saturates emphasize that above a given flatness, all of them are recognized by the labeling method. Smaller bubbles are artifacts that have no real counterpart. Their number increases with the flatness and their occurrence will be discussed below. The important result is that artifacts can be recognized and filtered out by using a volume threshold well below the volume of real bubbles. We have thus developed an objective determination of the flatness criterion in order to label correctly the bubbles in our foams: the flatness chosen has to be larger than this threshold. We chose 0.375 to prevent too many artificial seeds.

2. Identify a high GGW criterion $\left(T_{\text {growth }}\right)$ for growing seeds.

From its definition, $T_{\text {growth }}$ could potentially range between 0 (complete filling of every interconnected space) and 1 (no growth). $T_{\text {growth }}$ was adjusted so that bubbles grow up to approximately $80 \%$ of their final volume. A value of 0.9 was taken to satisfy this criterion. This enabled us to eliminate all potential artificial seeds present in the middle of the final bubbles while avoiding intruding bubbles fig 3a,4c). Smaller artificial seeds could still potentially be present (we mostly find them close to the vertices or close to the edges of the 3D image), but are easily eliminated using a volume threshold (see above). Smaller value of $T_{\text {growth }}$ are not advised since they could lead to invasion of other bubbles and lead to unphysical very anisotropic bubbles (see figure 3a).

3. Remove any artificial seeds.

The former step is performed starting with the seed with the highest distance and iterating through all seeds 
in a descending manner. For each seed, we check whether it overlaps with an existing grown bubble. The seed is kept and allowed to grow if the overlap is less than $40 \%$ by volume (the exact value is not restrictive). This step is repeated until all seeds have been processed. After this step, the number of final bubbles is fixed.

4. Identify a low GGW criterion $\left(T_{\text {fill }}\right)$ for filling bubbles.

0.3 was found as the best compromise between growing bubbles (lower value) and overlapping bubbles (higher value) in all the systems examined. The parameter while important for visual inspection and a reconstruction of the film areas, played only a very minor role on the final quantitative results (bubble count, anisotropy, face count, volume, etc)

After the image analysis, using the set of parameters Flatness $=0.375, T_{\text {growth }}=0.9$ and $T_{\text {fill }}=0.3$, we found that the labeled FOAM-M contains 400 bubbles with a mean volume $\mathrm{V}=0.08 \pm 0.02 \mathrm{~mm}^{3}$. Moreover, we can evaluate the liquid fraction from the PLAT images as the ratio of the Plateau border network volume to the total volume of the image; for FOAM-M, this is $7.2 \%$. We have verified that this measurement of the liquid fraction is not very sensitive to the threshold level applied to the raw reconstruction images.

\section{COMPARISON TO PREVIOUS METHOD}

To demonstrate the robustness and reusability of the technique, we use the same set of parameters established in the previous section to label a very different foam sample, FOAM-P which corresponds to a highly polydisperse, disordered foam. We verified by direct visual inspection on various slices that these parameters resulted in a good labeling.

In order to completely validate our labeling technique, we compared our method to the current state of the art. We thus independently filtered, segmented, labeled, and analyzed the same foam sample, FOAM-P, using an approved, existing method recently developed $[6,8]$ and based on a commercial software package.

We show in Figure 6c,d the histograms of the bubble size and face counts for the poly-disperse FOAM-P, using the two different procedures. We observe that the two different segmentation and labeling techniques give similar results, providing, for the existing (Lambert) and current (this manuscript) methods respectively: a bubble count of 6191 and 6299 , with an volume of $0.08 \pm 0.12 \mathrm{~mm}^{3}$ and $0.09 \pm 0.14$ and face count $9.9 \pm 4.7$ and $11.1 \pm 4.4$.

We also performed an individual comparison by matching each bubbles between the two methods (smallest distance criterion) and comparing their respective volume and face count (Figure 6cd). Again, the results are very good, emphasized by the large number of bubbles matched between the two methods, and allows to conclude that such results validate clearly our new labeling method. For this foam, we estimate the average liquid fraction to be $8.3 \%$ (we note the liquid fraction varies slightly in the vertical direction ranging from $7.0 \%$ near the top of the image to $9.4 \%$ at the bottom).

\section{DISCUSSION}

\section{Parameter Sensitivity}

As mentioned earlier, a visual inspection is of paramount importance to test the effects of the two parameters Flatness and $T_{\text {growth }}$ and to ensure an accurate labeling of the bubbles. None the less, we performed a thorough analysis by varying systematically both parameters to give some insights about their acceptable range. In this analysis $T_{\text {fill }}$ was fixed to 0.3. Results are displayed in figure 4. As discussed previously, number of large bubbles does not change significantly for a flatness between 0.3 and 0.5 , while the number of artificial bubbles increases with the flatness, without being strongly affected by $T_{\text {growth }}$. While $T_{\text {growth }}$ s effect on bubble count and volume was only significant at a value above 0.95 , a value below 0.8 caused bubbles to start to grow inside of each other (so-called intruding bubbles). Intruding bubbles is most directly seen in topology, but anisotropy is also affected.

It is important to emphasize that both parameters are dimensionless. Therefore, approximately the same value could be used in future studies irrespective of the bubble sizes. Dealing with segmented images with a different quality, parameters value would need to be slightly adapted since noisier data would lead to more artificial seeds, that would need to be eliminated by decreasing slightly Flatness and / or adjusting the threshold for the minimal accepted bubble size (10,000 voxels). We expect that the lower the signal to noise the larger this minimal accepted bubble size would need to be and for perfect images (e.g. in silico data) the requirement could be removed entirely. In any case, 
we would advise careful visual inspection and following the optimization procedure so as to ensure the quality of the bubble labelling.

\section{Artifacts}

The analysis performed above emphasizes that artifacts can be simply filtered out a posteri since their size is significantly smaller than the physically relevant bubbles. Artifacts are present in any labeling method and arise from a number of sources but primarily from the quality of the initial segmented image including truncation effects when Plateau border were near the edge of the image. The way the labelling procedure proceeds, from deeper local minima to smaller one, allows the growth of the most physically relevant bubbles first, reducing less space for the artifacts. As emphasized above, it is important to note that the two quantities that select the number of final bubbles, namely Flatness and $T_{\text {growth }}$, are dimensionless numbers. It means a priori that the criteria applied here are not connected to any physical length scales and the same procedure and parameter selection should hold for other foams with any bubble sizes and roughly the same liquid fraction, without adding more artifacts. In fact, this was checked here by using the same parameters to reconstruct successively both FOAM-M and FOAM-P.

\section{Filling and Final Steps}

The final steps involving filling with $T_{\text {fill }}$ and the Voronoi tesselation are important for the final image and particularly for the film reconstruction. We applied the Voronoi tesselation in all of our analyses to ensure accurate volumes as the MASK should consist $100 \%$ of bubbles. However, we note in images with empty space in the MASK image not corresponding to bubbles this extra step could drastically reduce the quality of the results as bubbles would grow unconstrained into the entire MASK volume (similar to figure 3a) possibily effecting the reliability of face count, anisotropy, and volume.

\section{Morphological Considerations}

The technique we presented did not utilize the physical mechanisms involved in bubble morphology or film shape. Rather, films are reconstructed by the dilation/growing of two neighboring bubbles, guided by the distance map. The ordering of the procedure means that larger bubbles will have first priority to fill this region with small bubbles coming later. This procedure may lead to deviations from the Plateau rules obeyed by dry foams [1] and consequently potentially unphysical films. Therefore we, at this stage, make no attempt to investigate the exact shape of the films connection at the Plateau Borders and vertices. We can imagine that the addition of physical constraints to the GGW method and a fine tuning of the $T_{\text {fill }}$ and voronoi steps would provide a more accurate reconstruction near the Plateau Borders and consequently more physically meaningful films. Alternatively an iterative or optimization approach could be developed by combining this method with a relaxation step.

\section{CONCLUSIONS}

In this manuscript, we have presented a new, generic and open method, which has been validated by successively labeling thousands of bubbles inside samples of liquid foams. The Gradient Guided Watershed procedure provides a reliable tool to label bubbles based on physical arguments that are explicitly controlled by the user and not hidden inside a commercial software page. The validation has been performed on highly disordered real foams. Especially the comparison with an already published method (based on a commercial software) was found to yield an excellent agreement. More generally, we claim that our method can be applied to any other multiphasic and cellular material as long as the cellular network can be imaged and segmented. This should provide a powerful tool for both the X-ray tomography and Materials communities.

The labeling and segmenting method we used provides a leap in the field of 3D foam analysis. The completely open nature of our algorithm [17] and the tools used to reproduce the results means that the only limits for such analyses are hardware and processing power. Additionally with the open and free choice of tools for analysis the potential to advance the field with new metrics for bubble shape and organization, network and topological analysis, and longitudinal studies are drastically increased. We also allow multiple groups working with multiple different 
instruments and analysis tools to perform identical labeling and thus avoiding systematic differences on the postprocessing side. Finally this should allow researchers to further collaborate and share data, ideally culminating in a large centralized database of 3D foams, which could be used to test and verify new theories about the behavior and fundamental physics of foams.

If bubbles tracking is now possible inside resting or slowly evolving foams (such as coarsening), it raises the question of the feasibility of such an approach for moving bubbles inside 3D foam flows and dynamical evolution on relatively faster scales. It is especially encouraging that these analysis tools are performing very well on the data sets acquired with an unprecedented sub-second temporal resolution. The high-throughput, hands-free processing made possible with our tools means that the previously unfathomably daunting task of analyzing thousands of bubbles in hundreds of measurements is now a simple matter of computational time. This performance allows to conclude that all the tools are now available for the assessment of the foam dynamics in 3D, which requires many bubbles and measurements for a clear quantification of the behavior and mechanical properties in the system.

\section{ACKNOWLEDGEMENTS}

Stéphane Santucci, Benjamin Dollet and Christophe Raufaste thank the GDR 2983 Mousses (CNRS) for supporting travel expenses. Kevin Mader acknowledges financial support from the National Competence Center for Biomedical Imaging in Lausanne, Switzerland.

[1] D. Weaire, S. Hutzler, The Physics of Foams, Oxford University press, Oxford, 2000.

[2] I. Cantat, S. Cohen-Addad, F. Elias, F. Graner, R. Höhler, O. Pitois, F. Rouyer, Saint-Jalmes A., Les mousses, structure et dynamique, Berlin, Paris, 2010.

[3] C. P. Gonatas, J. S. Leigh, A. G. Yodh, J. A. Glazier, B. Prause, Magnetic resonance images of coarsening inside a foam., Physical Review Letters 75 (1995) 573-576.

[4] C. Monnereau, M. Vignes-Adler, Dynamics of 3D Real Foam Coarsening, Physical Review Letters 80 (1998) 5228-5231.

[5] J. Lambert, I. Cantata, R. Delannay, A. Renault, F. Graner, J. A. Glazier, I. Veretennikov, P. Cloetens, Extraction of relevant physical parameters from 3D images of foams obtained by X-ray tomography, Colloids and surfaces. A, Physicochemical and engineering aspects 263 (2005) 295-302.

[6] J. Lambert, R. Mokso, I. Cantat, P. Cloetens, J. A. Glazier, F. Graner, R. Delannay, Coarsening foams robustly reach a self-similar growth regime, Phys. Rev. Lett. 104 (2010) 248-304.

[7] R. Mokso, F. Marone, M. Stampanoni, Real-Time Tomography at the Swiss Light Source, in: AIP Conf. Proc., SRI2009, 2009 .

[8] J. Lambert, I. Cantat, R. Delannay, R. Mokso, P. Cloetens, J. A. Glazier, F. Graner, Experimental growth law for bubbles in a moderately "wet" 3D liquid foam., Physical Review Letters 99 (2007) 058304.

[9] A. J. Meagher, M. Mukherjee, D. Weaire, S. Hutzler, J. Banhart, F. Garcia-Moreno, Analysis of the internal structure of monodisperse liquid foams by X-ray tomography, Soft Matter 7 (2011) 9881.

[10] M. Saadatfar, A. P. Sheppard, T. J. Senden, A. J. Kabla, Mapping forces in a 3D elastic assembly of grains, Journal of the Mechanics and Physics of Solids 60 (2012) 55-66.

[11] G. Lin, U. Adiga, K. Olson, J. F. Guzowski, C. A. Barnes, B. Roysam, A hybrid 3D watershed algorithm incorporating gradient cues and object models for automatic segmentation of nuclei in confocal image stacks., Cytometry. Part A : the journal of the International Society for Analytical Cytology 56 (2003) 23-36.

[12] F. Meyer, Topographic distance and watershed lines, Signal Processing 38 (1994) 113-125.

[13] K. Golemanov, N. D. Denkov, S. Tcholakova, M. Vethamuthu, A. Lips, Surfactant mixtures for control of bubble surface mobility in foam studies., Langmuir The Acs Journal Of Surfaces And Colloids 24 (2008) 9956-9961.

[14] M. Stampanoni, F. Marone, P. Modregger, B. R. Pinzer, T. Thüring, J. Vila-Comamala, C. David, R. Mokso, Tomographic Hard X-ray Phase Contrast Micro-and Nano-imaging at TOMCAT, in: AIP Conference Proceedings, volume 1266 , p. 13.

[15] F. Marone, B. Münch, Fast reconstruction algorithm dealing with tomography artifacts, Proceedings of SPIE (2010).

[16] I. Ragnemalm, The Euclidean distance transform in arbitrary dimensions, Pattern Recognition Letters 14 (1993) 883-888.

[17] http://code.google.com/p/distance-guided-labeling/ 


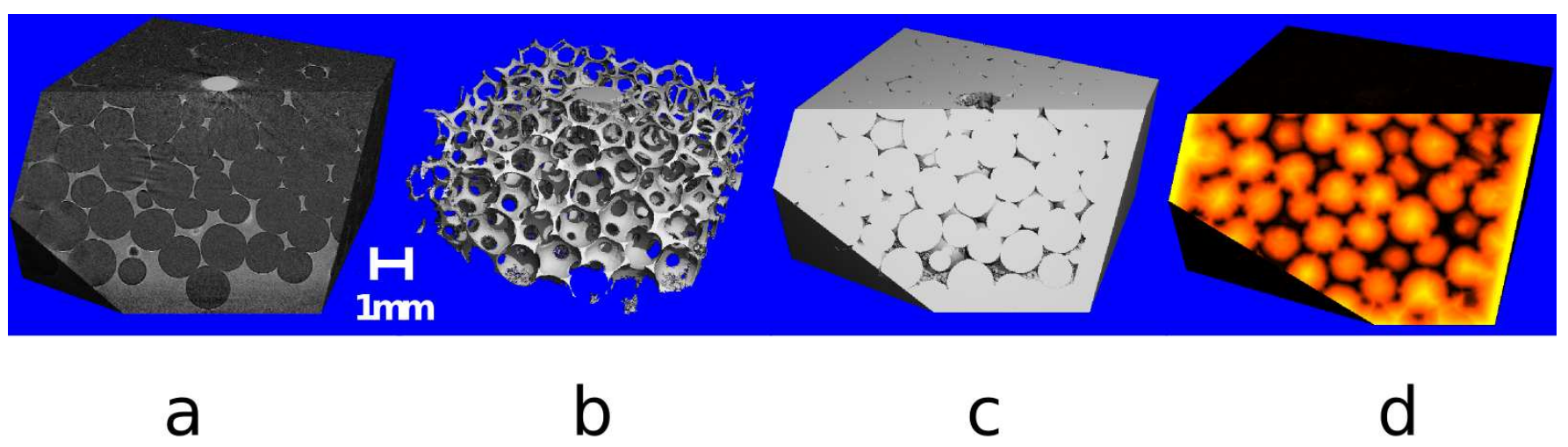

FIG. 1. Foam Imaging. We show in this figure the steps involved for foam imaging using X-Ray tomography. (a) Reconstructed absorption values from the sample. The liquid in the Plateau borders (gray) absorbs much more than the air within the bubbles (black). (b) Segmented Plateau borders. (c) Inverse of (b), showing the bubbles of the image. (d) Distance map created from the inverse of the Plateau borders. It shows how far each voxel in the foam is away from the nearest Plateau border. Yellow regions are far away and black regions are closer.

\section{FIGURES}




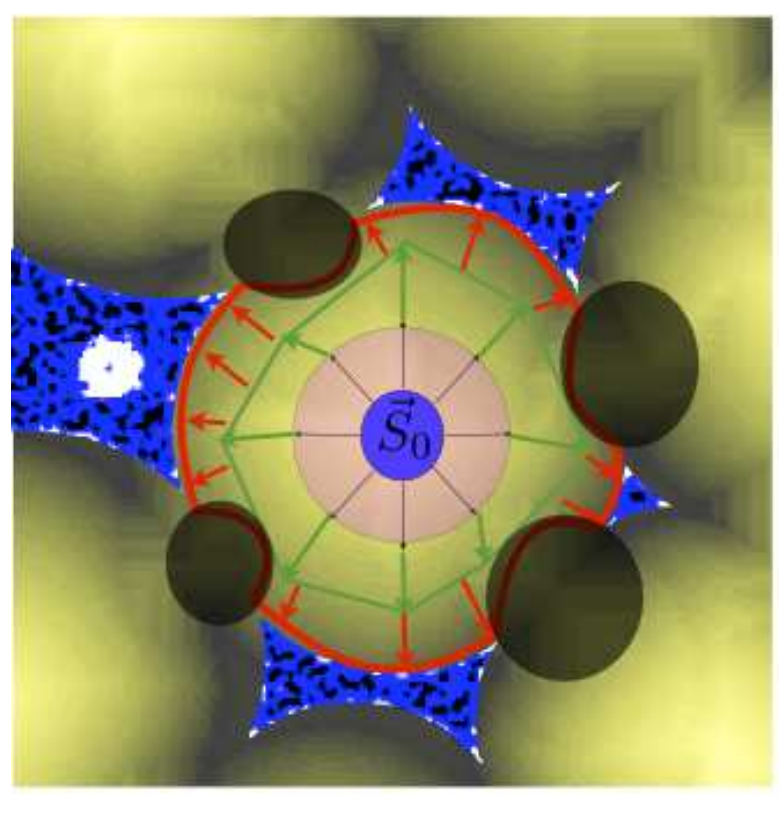

a

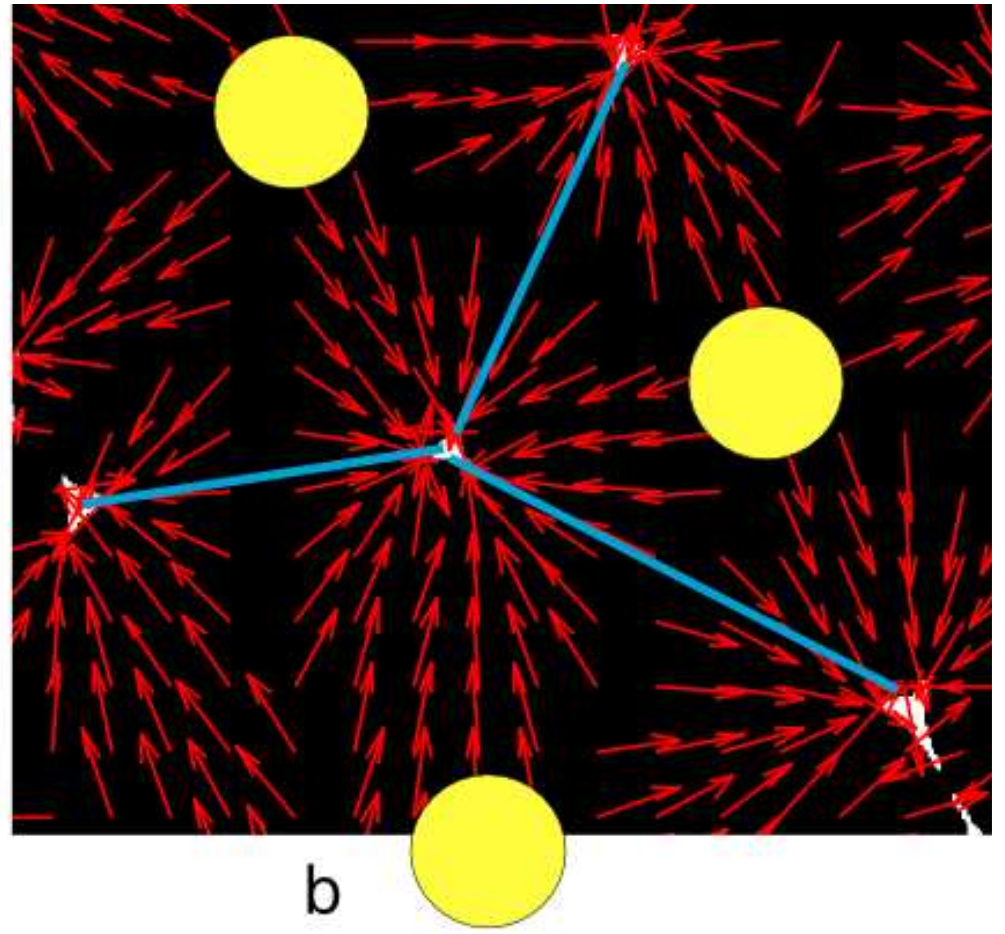

FIG. 2. Gradient Guided Watershed. The principle is shown through these two 2D images. (a) shows a local maximum selected to grow as a bubble $(S 0)$ and the surrounding Plateau borders (blue) and distance map colored in yellow. The progress of the bubble is shown as arrows through several iterations. The first few (light red) are isotropic as the distance map increases uniformly from local maximum. The next (green) become slightly more anisotropic with regions no longer in-line with the proximal Plateau borders growing more slowly. The final step (dark thick red) is very anisotropic with no growth in the black circle regions and strong growth to the Plateau borders. The figure also illustrates how poorly constrained the bubbles are by the plateau borders in lower liquid fraction, open cellular materials. (b) shows three local maxima (yellow circles), Plateau borders (white triangles), and the distance map as a gradient field (red arrows). The growth of each local maxima would follow the arrows toward the Plateau borders. 


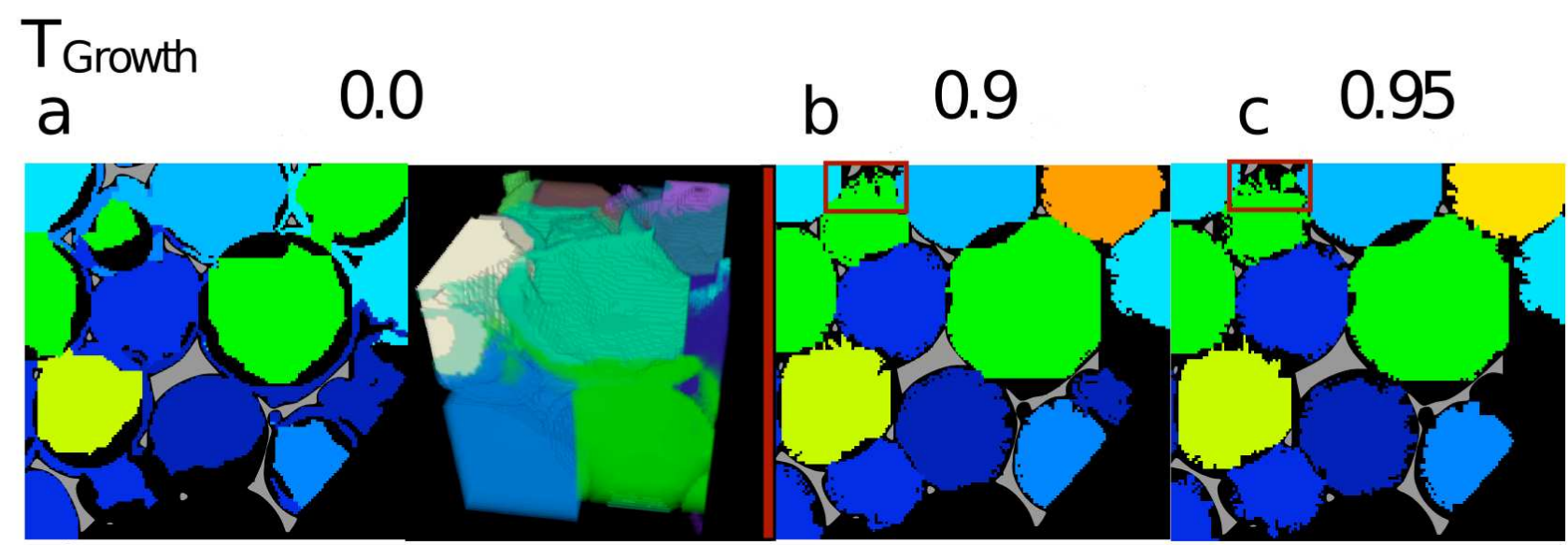

Flatness

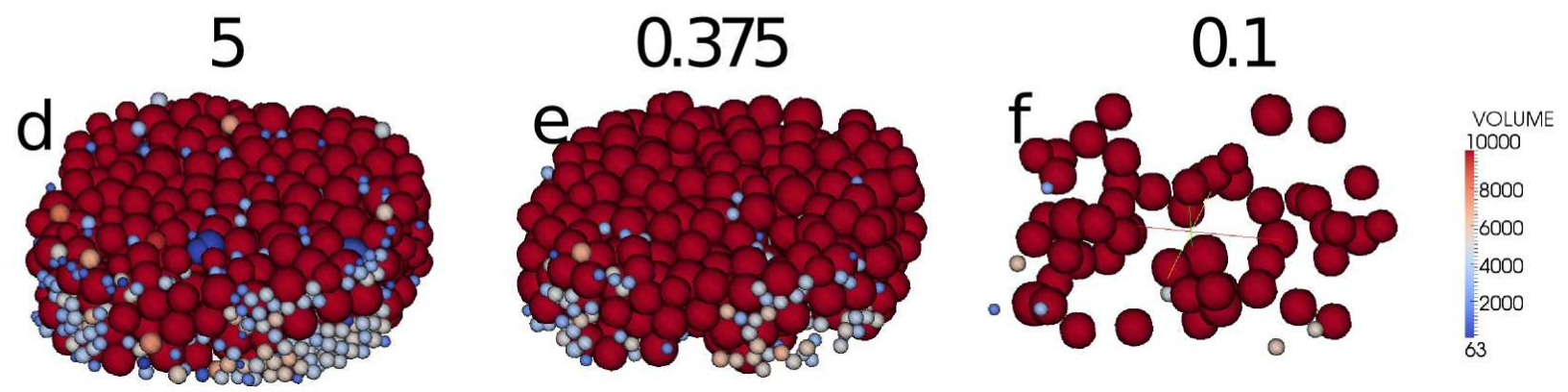

FIG. 3. Illustration of the effect of the parameters $T_{\text {growth }}$ and Flatness on the labeling of bubbles. (a-c) show 3 different values of $T_{\text {growth }}$ and the resulting labeling in a small region. The plateau border is colored white. (a) Shows a too low threshold such that the bubbles creep or invade neighboring bubbles shown in a slice and 3D illustration. (b) Shows the value, which was used for the analysis of FOAM-M and FOAM-P with nearly full bubbles and little to no creep. (c) shows a $T_{\text {growth }}$ value, which is too high and prevents the bubbles from sufficiently filling the cavities and then during $T_{\text {fill }}$ overlap. Specifically the bubble in the red box is not completely filled in and could result in bubbles invaginating each other. (d-f) Show 3 different values for Flatness and the labeled bubbles drawn as spheres colored by the volume between 0 and 10,000 voxels. (d) shows a value, which is too high and results in a large number of very small bubbles. (e) Shows the value used for the analysis of FOAM-M and FOAM-P. (f) Shows a too low value for Flatness resulting in too few seeds, which consequently become bubbles. 
a

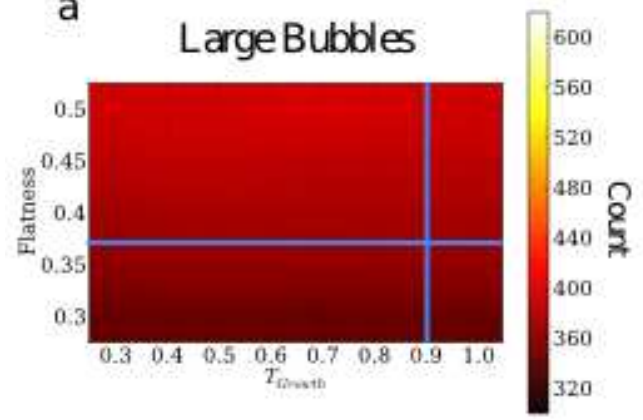

b
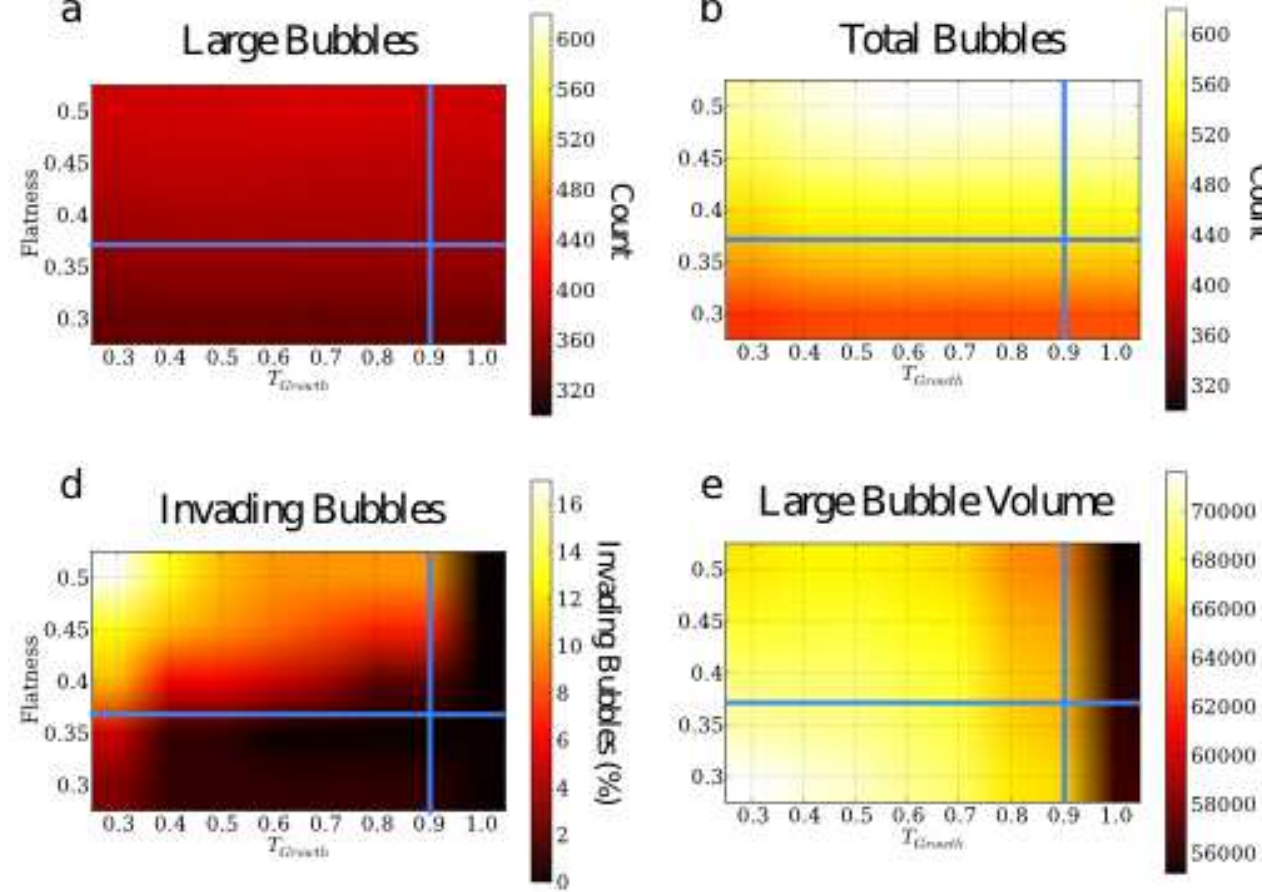

e Large BubbleVolume

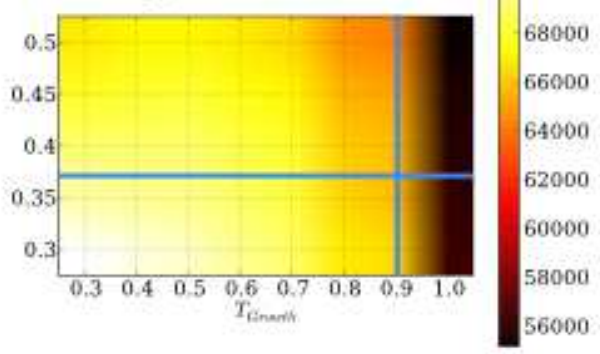

C

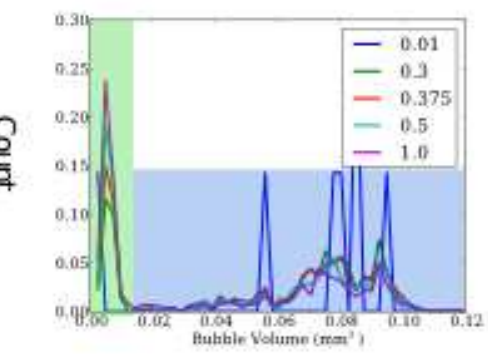

$f$

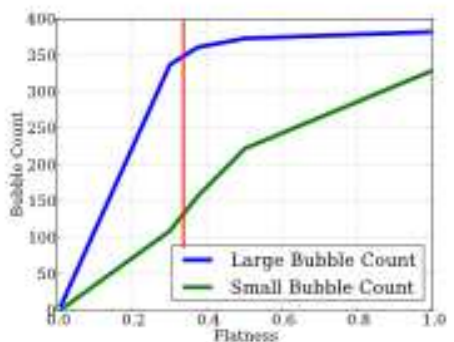

FIG. 4. Quantitative sensitive analysis of the parameters $T_{\text {growth }}$ and Flatness on the labeling of bubbles using the FOAM-M system. In a,b,d, and f, Flatness is shown on the X-axis and $T_{\text {growth }}$ on the $\mathrm{Y}$-axis. The parameters are varied around the selected values with $T_{\text {growth }}$ going all the way to $T_{\text {fill }}$. The point where the blue lines indicate the value used for the analysis of FOAM-M and FOAM-P. (a) Shows the number of large ( $>10,000$ voxels) bubbles. (b) Shows the number of total bubbles. (c) Shows the volume distribution (x-axis) against probability (y-axis) of bubbles based on only the Flatness (colored lines) parameter with $T_{\text {growth }}$ fixed at 0.9 . (d) Shows the percentage of bubbles which invading or creep bubbles assessed by counting the number of bubbles with more than 20 faces. (e) Shows the mean volume of bubbles, which contain more than 10,000 voxels (f) Shows the bubble count for large and small bubbles against the Flatness parameter with a fixed $T_{\text {growth }}$ of 0.9 . The definitions of large and small are indicated by the blue and green colored regions respectively in (c) 

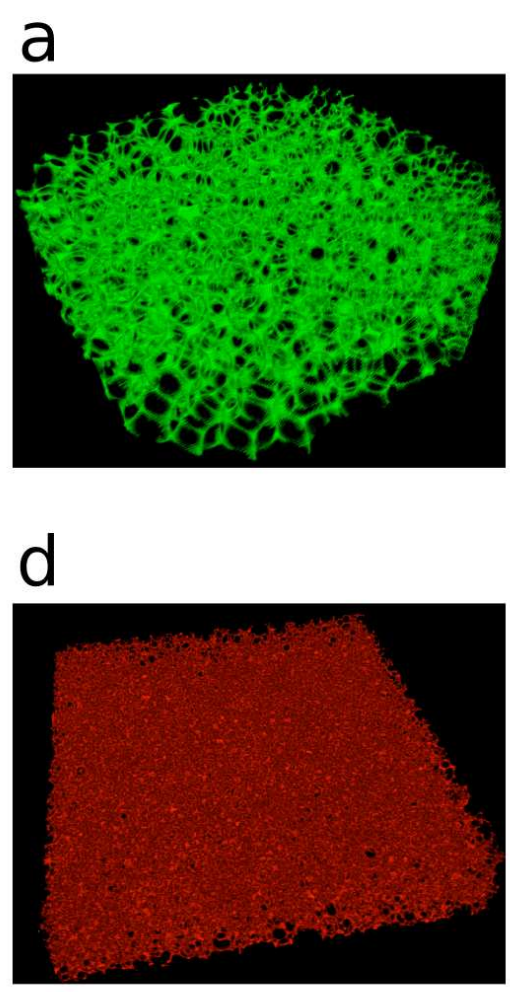

b
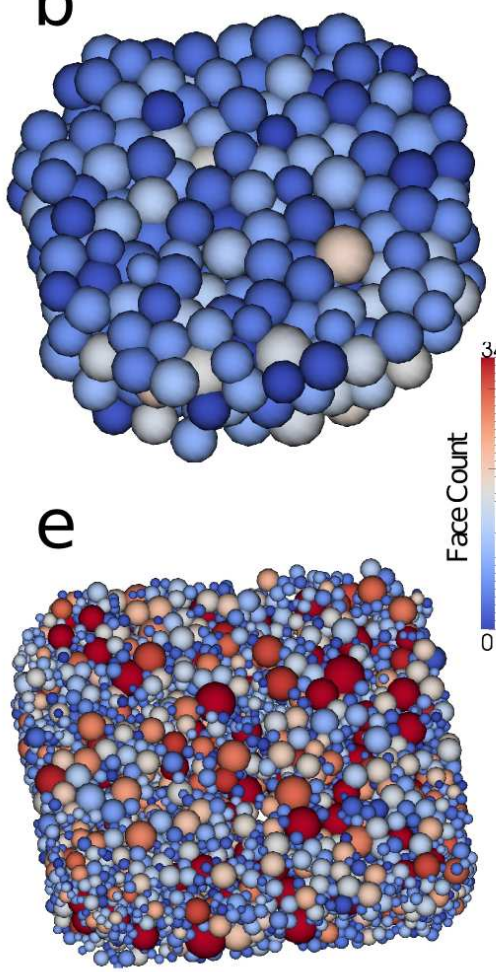

\section{C}

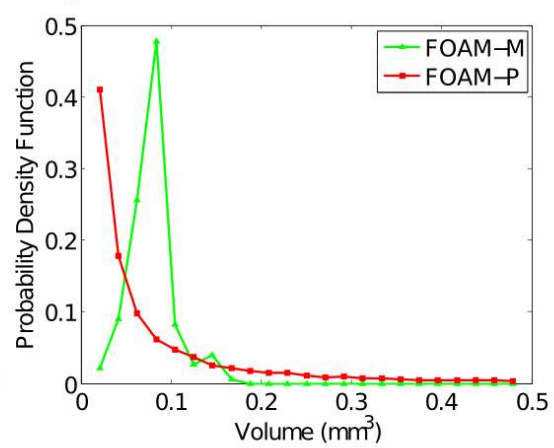

20

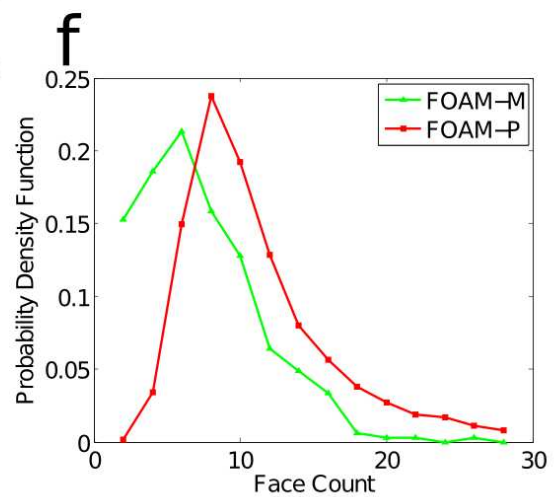

FIG. 5. Reconstruction and labeling of two different foams using the same Flatness $=0.375$ and $T_{\text {Growth }}=0.9$. The foams examined were FOAM-P (poly-dispersive foam) and the FOAM-M (monodisperse foam) with (a) and (d) showing the segmented Plateau borders for the two foams. (b) and (e) show the labeled bubbles colored by the number of facets the given bubble has. (c) shows the histogram of bubble volume with number fraction plotted on the $\mathrm{Y}$-axis and bubble volume plotted on the $\mathrm{X}$-axis. Number fraction indicates the number of bubbles with this volume over the total number of bubbles. The standard-deviation of the FOAM-P distribution is 7 times larger than FOAM-M. (f) shows the histogram for face count with the Y-axis being number fraction. 

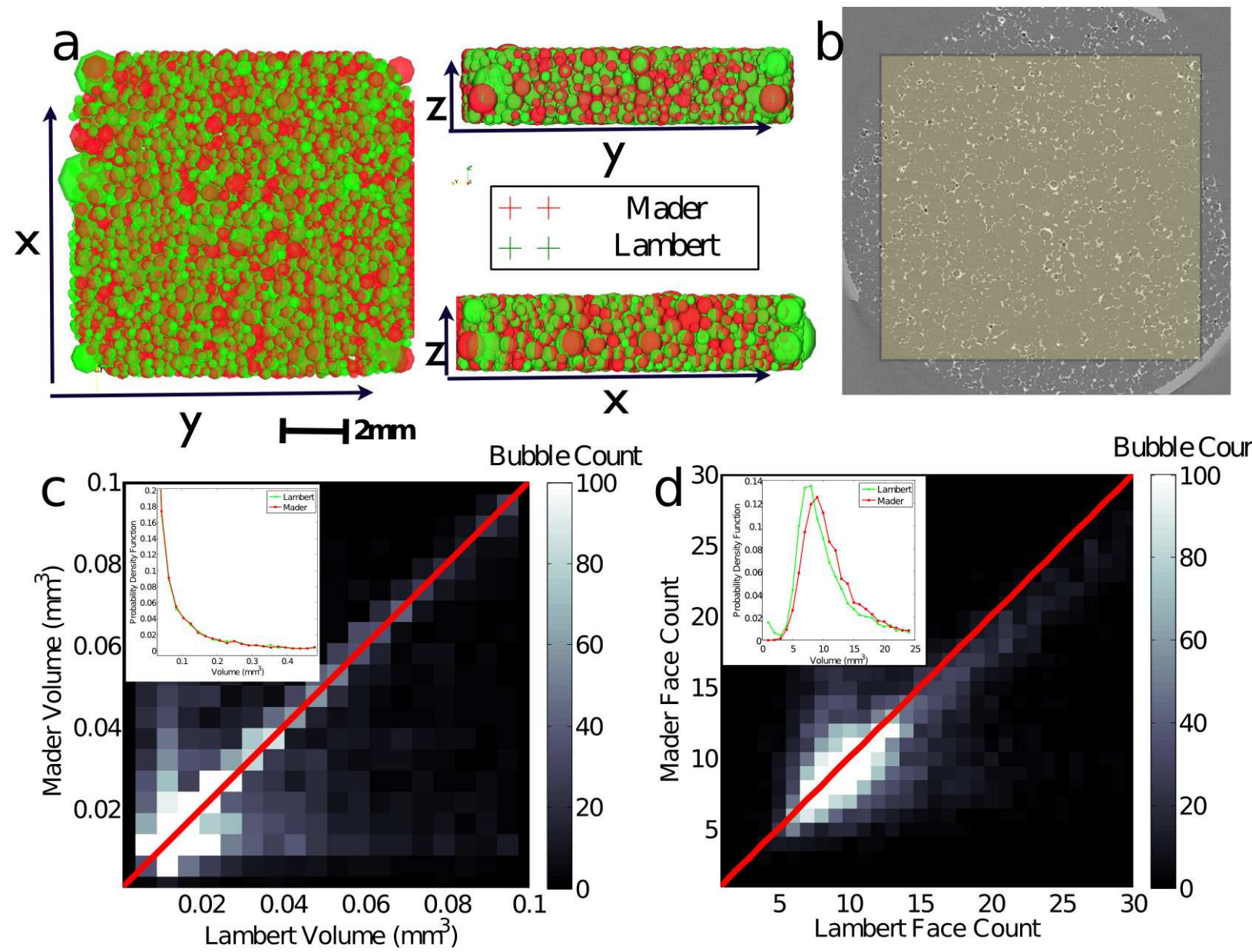

FIG. 6. Comparison between the techniques developed in this paper (red) and those used in [8] (green) using the FOAM-P measurement analyzed with both techniques. (a) shows the visual comparison with red spheres being bubbles coming from the tools we developed and green spheres being bubbles from the former paper, [8]. (b) shows an XY slice taken from the absorption data with the region of interest compared highlighted (c and d) show comparison of bubbles matched between the two methods. The red line in both images indicates a perfect matching between the methods (c) shows a $2 \mathrm{D}$ histogram of the volume. The $\mathrm{X}$-axis shows the volume in the Lambert labeling and the Y-axis shows the volume from the labeling introduced in the manuscript. The color indicates the number of bubbles with black being 0 and white being more than 100 . The volume axis in this graph is limited due to the sparsity of bubbles larger than 0.1 where agreement was also good (not shown). The inset shows two volume distributions plotted against each other. The two distributions seem to match very well in shape, mean, and standard deviation. (d) shows a 2D histogram of the face count. The X-axis shows the face count in the Lambert labeling and the $\mathrm{Y}$-axis shows the face count from the labeling introduced in the manuscript. The color indicates the number of bubbles with black being 0 and white being more than 100. The inset shows the face count distribution plotted on a linear scale. The mean faces numbers equal to $9.9 \pm 4.7$ (Lambert) and $11.1 \pm 4.4$ (Mader). 